\title{
Definición de infraestructuras verdes: una propuesta metodológica integrada mediante análisis espacial*
}

\author{
Francisco Aguilera Benavente \\ Víctor Manuel Rodríguez Espinosa \\ Montserrat Gómez Delgado \\ Universidad de Alcalá. Departamento de Geología, Geografía y Medio Ambiente \\ f.aguilera@uah.es \\ victor.rodriguez@uah.es \\ montserrat.gomez@uah.es
}

\section{Resumen}

El concepto de Infraestructura Verde (IV) ha comenzado a ocupar un lugar destacado en la planificación ambiental desde finales del siglo XX. Con un carácter eminentemente espacial, se plantea la necesidad de buscar procedimientos que permitan, de alguna forma, concretar estas infraestructuras a partir de la evaluación y cartografiado de las dimensiones que la integran. En el presente trabajo se propone una metodología de análisis espacial (mediante Sistemas de Información Geográfica) que pretende ayudar a concretar su delimitación y cartografía a partir del análisis en cada punto del territorio de cuatro dimensiones asociadas a la IV: la contribución a los servicios ecosistémicos de diversos elementos territoriales, la conectividad ecológica, la accesibilidad o permeabilidad a la población y el valor ecológico. A pesar de las limitaciones, se trata de una propuesta suficientemente concreta, pero a la vez abierta, y aplicable en cualquier área de estudio, lo que podría facilitar la implementación de IV al proceso de planificación en España.

Palabras clave: infraestructuras verdes; Sistemas de Información Geográfica; servicios ecosistémicos; conectividad ecológica; valor ecológico

* Trabajo integrado en el proyecto «Instrumentos de Geosimulación y planificación ambiental en la ordenación territorial de ámbitos metropolitanos. Aplicación a escalas intermedias SIMURBAN2» (Ref. CSO2012-38158-C02-01), financiado por el Ministerio de Economía y Competitividad (Secretaría de Estado de Investigación, Desarrollo e Innovación). 
Resum. Definició d'infraestructures verdes: una proposta metodologica integrada a través d'una anàlisi espacial

El concepte d'Infraestructura Verda (IV) ha començat a ocupar un lloc destacat en la planificació ambiental des de finals del segle XX. Amb un caràcter eminentment espacial, es planteja la necessitat de buscar procediments que permetin d'alguna manera concretar aquestes infraestructures, a partir de l'avaluació i el cartografiat de les dimensions que la integren. En aquest treball proposem una metodologia d'anàlisi espacial (mitjançant Sistemes d'Informació Geogràfica), amb l'objectiu de concretar-ne la delimitació i la cartografia a partir de l'anàlisi en cada punt del territori de quatre dimensions associades a la IV: la contribució als serveis ecosistèmics de diversos elements territorials, la connectivitat ecològica, l'accessibilitat o permeabilitat a la població i el valor ecològic. Malgrat les limitacions, es tracta d'una proposta suficientment concreta, però oberta a la vegada, i aplicable a qualsevol àrea d'estudi, fet que podria facilitar la implementació de IV al procés de planificació espanyol.

Paraules clau: infraestructures verdes; Sistemes d'Informació Geogràfica; serveis ecosistèmics; connectivitat ecològica; valor ecològic

Résumé. Définition de l'infrastructure verte : une proposition méthodologique intégrée grâce à l'analyse spatiale

Le concept d'Infrastructure Verte (IV) commence à prendre de l'importance dans la planification environnementale dès la fin du XXe siècle. Avec un caractère éminemment spatial, elle envisage le besoin de chercher des procédés permettant d'une certaine façon la concrétisation de ces infrastructures. Le présent travail propose une méthodologie d'analyse spatiale (au moyen de Systèmes d'Information Géographique), dont le but est d'aider à concrétiser la délimitation et la cartographie à partir de l'analyse de quatre dimensions associées à l'IV sur chaque point du territoire : la contribution aux services écosystémiques d'éléments territoriaux divers, la connectivité écologique, l'accessibilité ou la perméabilité de la population et la valeur écologique. Malgré ses limites, il s'agit d'une proposition suffisamment concrète, mais à la fois ouverte, et pouvant s'appliquer à n'importe quel domaine d'études, ce qui pourrait faciliter l'application de l'IV au processus de planification en Espagne.

Mots-clés: Infrastructure verte; Systèmes d'Information Géographique; services écosystémiques; connectivité écologique; valeur écologique

\section{Abstract. Defining Green Infrastructure: Integrated methodological proposal using spatial analysis}

Since the late twentieth century, the concept of Green Infrastructure (GI) has begun to occupy a prominent place in environmental planning. With particular regard to space, there is a growing need to develop procedures which enable identifying GI based on evaluating and mapping its dimensions. This paper proposes a spatial analysis method using geographical information systems in order to demarcate and map GI by analyzing at each point of the territory the following four GI-associated dimensions: the contribution to ecosystem services of various territorial elements, ecological connectivity, accessibility or permeability to the population, and ecological value. Despite its limitations, the proposal is sufficiently well-defined, but also open and applicable to any field of study, which could facilitate the implementation of GI in Spanish planning.

Keywords: Green Infrastructure; geographic information systems; ecosystem services; ecological connectivity; ecological value 


\section{Sumario}
1. Introducción
4. Discusión y conclusiones
2. Datos de partida y área de estudio
Referencias bibliográficas

3. La propuesta metodológica

\section{Introducción}

El concepto de Infraestructura Verde (IV) ha comenzado a ocupar un lugar destacado en la planificación ambiental desde finales del siglo XX. Entendida como una red de espacios verdes interconectados, planificados y gestionados de acuerdo a sus valores ecológicos (Benedict y McMahon, 2006); como una red multifuncional de espacios verdes, urbanos y rurales, nuevos o ya existentes, capaces de prestar un amplio abanico de beneficios ambientales y calidad de vida a la comunidad (LUC, 2009; TCPA, 2012; Nature England, 2009) o como «una red de zonas naturales y seminaturales y de otros elementos ambientales, planificada de forma estratégica, diseñada y gestionada para la prestación de una extensa gama de servicios ecosistémicos» (EC, 2013:3) (más definiciones del concepto se pueden encontrar en EEA, 2011). Así, el concepto de IV está asociado a diferentes aspectos o dimensiones: i) multifuncionalidad, ya que integra elementos territoriales que pueden proporcionar muchos y variados bienes y servicios (Sbara et al., 2007; CE, 2010; DGMA, 2011; Remolina, 2011; CE, 2013); ii) conectividad ecológica, pues constituye una red de zonas naturales y seminaturales y otros elementos ambientales (EC, 2013:3); iii) conservación, ya que fomenta la conservación de ecosistemas naturales valiosos y la preservación de la biodiversidad; iv) prestación de servicios a la población, entre ellos, y sobre todo, servicios ecosistémicos, entendidos como beneficios potenciales que, activa o pasivamente, la sociedad obtiene de los ecosistemas y que se concretan en servicios reales una vez que son demandados, usados o disfrutados (Fisher et al., 2009; Corredor et al., 2012); y v) la multiescalaridad, puesto que se articula a diferentes escalas, desde la supranacional a la local (TCPA, 2012).

Así, la IV, con un carácter eminentemente espacial y con clara vinculación a la ordenación del territorio, surge con la intención de ofrecer un instrumento nuevo y eficaz para guiar un desarrollo (territorial, urbanístico, económico, etc.) acorde a los principios de sostenibilidad (Mwirigi et al., 2012; Tzoulas et al., 2007), incidiendo en las ideas de red y servicio. Tiene así vocación de constituirse en una más de las infraestructuras de servicios que aseguran el funcionamiento de los territorios y atienden las necesidades de cualquier sociedad, pudiendo constituir al mismo tiempo una oportunidad para «repensar» las relaciones de los entornos urbanos con el medio (Mell, 2012).

Lo atractivo del concepto y su vinculación con la planificación y ordenación del territorio ha provocado que las IV se hayan ido incorporando de forma progresiva y cada vez más evidente en políticas, planes, actuaciones y experiencias territoriales, a diferentes escalas (Benedict y McMahon, 2006; Rodríguez y Agui- 
lera, 2016), poniendo de manifiesto su innegable potencial como instrumento de apoyo y orientación para una planificación territorial más sostenible.

Sin embargo, la progresiva incorporación de la IV al planeamiento de muchos países del entorno europeo, incluido España (Rodríguez y Aguilera, 2016), hace cada vez más necesario el desarrollo de herramientas que ayuden a planificadores, agentes y administración, por ejemplo, a elaborar planes de carácter territorial y a identificar y cartografiar elementos o áreas candidatas a incorporarse en la misma.

A este respecto, algunas normativas a nivel europeo y, en menor medida, alguno de los planes territoriales o sectoriales españoles, como el Plan de Infraestructura Verde de la Comunidad Valenciana (CVOPVT, 2011), ofrecen indicaciones y recomendaciones generales para la definición y establecimiento de IV, aunque aún es necesario avanzar en esta cuestión (Rodríguez y Aguilera, 2016).

Si se tiene en cuenta que, superando la mera dimensión ecológica, las IV pueden considerarse instrumentos vinculados a la planificación ambiental, resulta conveniente contar con herramientas para la identificación y cartografía de dichas infraestructuras, partiendo desde las diferentes sensibilidades o vertientes de este planeamiento (Ndubisi, 2003), que se corresponden con dimensiones de la IV. Así, por ejemplo, Kopperoinen et al. (2014), mediante talleres y técnicas participativas con diferentes agentes, desarrollan un método de valoración de la IV basado fundamentalmente en la cartografía de servicios ecosistémicos. Por su parte, Kang y Kim (2015) optan por una metodología fundamentada en la ecología del paisaje; empleando un análisis morfológico de patrones espaciales (MSPA), basado en el análisis del tamaño, forma y conectividad de las teselas de usos de suelo y utilizando el programa Guidos del JRC (Vogt, 2016). En la misma línea, Liquete et al. (2015) centran su propuesta en una combinación del enfoque de los servicios ecosistémicos y la conectividad ecológica; primero valoran los servicios ecosistémicos asociados a los usos del suelo para identificar áreas núcleo en la IV y analizan, después, la conectividad entre ellas empleando algunas de las aplicaciones gratuitas más utilizadas en estudios similares (Guidos o Linkage Mapper Connectivity Analysis Software).

No obstante, las propuestas existentes no abordan la definición de la IV desde una perspectiva que incluya algunas de las dimensiones clave de la misma, como por ejemplo la accesibilidad de la población a estos elementos (vinculada con el acceso al disfrute de ciertos servicios ecosistémicos), o solo se centran en alguna/algunas de ellas de manera particular, siendo necesario avanzar en el desarrollo de propuestas integradas.

En este sentido, el objetivo de este artículo es desarrollar una propuesta metodológica que permita, de forma sencilla y replicable, valorar y cartografiar los elementos y espacios susceptibles de integrar una IV, que sirva de apoyo en las tareas prácticas de planificadores y decisores, es decir, con indicaciones espaciales y concretas sobre dónde desarrollar una IV. Para ello, mediante un análisis de aptitud (Landscape Suitability Analysis) realizado con SIG, se identificarán las áreas más adecuadas para integrarse en una IV atendiendo a las dimensiones de conectividad, valor del territorio, multifuncionalidad y 
prestación de servicios ecosistémicos. La valoración de dichas dimensiones y la posterior integración en la cartografía de aptitud para la IV se realiza recurriendo al uso de instrumentos propios de diversas corrientes o vertientes del planeamiento ecológico, como la ecología del paisaje, los servicios ecosistémicos, etc., así como al uso de técnicas de evaluación multicriterio (EMC) que permitan la integración de las diferentes dimensiones.

El artículo se organiza de la siguiente forma: el epígrafe 2 muestra los datos de partida y el área de estudio escogida para probar la metodología propuesta, que se detalla en profundidad en el epígrafe 3, donde se cartografían las dimensiones de valor de la IV y se integran mediante técnicas EMC. El epígrafe 4, finalmente, muestra la discusión y conclusiones del trabajo.

\section{Datos de partida y área de estudio}

La presente propuesta, con vocación de ser aplicable en el ámbito español, requiere del uso de tres conjuntos de datos de partida: i) datos de ocupación del suelo con un nivel de detalle suficiente para su uso en escalas supramunicipales (lo que descartaría la cartografía Corine Land Cover); ii) una base cartográfica con información suficientemente completa y detallada sobre espacios naturales protegidos, hidrografía, red viaria, etc.; iii) un modelo digital de elevaciones igualmente con el nivel de detalle suficiente. A este respecto, y con el objetivo de ser aplicable en cualquier comunidad autónoma, así como presentar escalas de trabajo similares (1:25.000), se ha optado por utilizar las siguientes fuentes:

- Base de datos de ocupación del suelo SIOSE (Sistema de Información sobre Ocupación del Suelo de España): constituye una base de datos de ocupación del suelo para toda España a escala de referencia 1:25.000, con varias fechas disponibles para su descarga (en función de la comunidad autónoma). Una de sus características principales es la de ser una base de datos multiparámetro, al poder asociar a cada tesela diversas clases de usos/ coberturas con diferentes porcentajes de ocupación, ya sean estas coberturas simples o compuestas (formadas como combinación de varias coberturas simples) (IGN, 2015).

De dicha base de datos es posible derivar mapas de uso y cobertura del suelo, a través de mapas de porcentajes de cobertura de un determinado tipo o mediante la categorización del mismo; para esta última existen algunas propuestas, como la de Cantarino (2013), que asignan a los polígonos aquellos usos o coberturas claramente predominantes en cada uno de ellos, siguiendo un modelo jerárquico similar al de Corine Land Cover (EEA, 2007), y que ofrece 44 categorías en su nivel 3.

A este respecto, para la presente propuesta se ha optado por realizar una categorización ad hoc de la información de SIOSE que ha permitido adaptar el número de categorías de leyenda a las necesidades de la propuesta. En caso de aplicar la metodología en comunidades autónomas que dispongan de cartografía de ocupación y uso de suelo más detallada o de carácter categórico 
(caso del Mapa de Usos y Coberturas Vegetales del Suelo de AndalucíaMUCVA 1:25.000, el Mapa de Cubiertas del suelo de Cataluña-MSCC 1:5.000 o el Mapa de hábitat, Vegetación actual y usos del suelo de Euskadi 1:10.000, por ejemplo) podría emplearse esta en sustitución de SIOSE. Otras alternativas para todo el estado español podrían ser el Mapa Forestal o el Mapa de cultivos y aprovechamientos, ambos a escala 1:50.000.

- Base topográfica nacional a escala 1:25.000 (BTN25): constituye el conjunto de datos geográficos vectoriales de referencia de más detalle que cubren homogéneamente todo el ámbito del Estado (IGN, 2016a). Con un total de casi 90 capas en formato vectorial shapefile (*.shp), contiene información topográfica, red viaria, espacios naturales protegidos, zonas urbanas, hidrografía, etc.

- Modelo digital de elevaciones (MDT25): se trata de un modelo digital del terreno a nivel del suelo con paso de malla de $25 \mathrm{~m}$, con la distribución oficial de hojas 1:25.000 (IGN, 2016b). Dependiendo de las escalas de trabajo, podría sustituirse por el MDT5, con una resolución mucho mayor. En este caso se ha utilizado el de $25 \mathrm{~m}$ y, dado que la propuesta se plantea en un entorno ráster, el conjunto de capas vectoriales anteriores se ha rasterizado con este tamaño de píxel.

La metodología que se describe en los siguientes apartados ha sido aplicada en el Corredor del Henares (figura 1), importante franja urbano-industrial

Figura 1. Localización del Corredor del Henares (Madrid-Guadalajara)

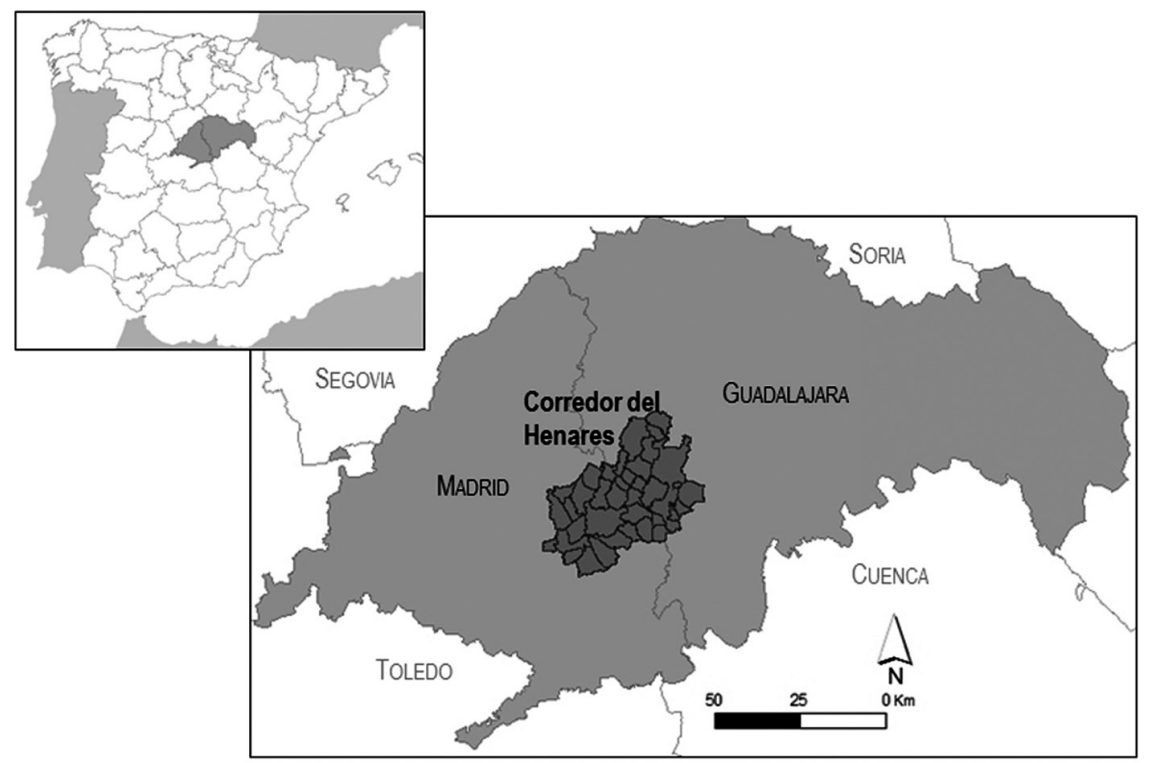

Fuente: elaboración propia. 
situada al noreste de la región urbana de Madrid y que se extiende desde la capital hasta el municipio de Guadalajara, a caballo entre dos comunidades autónomas diferentes (Madrid y Castilla-La Mancha); en total, 35 municipios que ocupan una superficie de más de 120 mil ha y en los que residen unos 750 mil habitantes.

En un ámbito como este resulta oportuno el diseño y conformación de una infraestructura de este tipo como una oportunidad para plantear un planeamiento estratégico (Rodríguez et al., 2015), pues, a pesar de haber experimentado en las últimas décadas un importante desarrollo urbanístico, todavía conserva amplios espacios de marcado componente rural, localizados, fundamentalmente, en los márgenes norte y sur del eje principal del corredor. Además, alberga múltiples espacios naturales protegidos bajo diversas figuras y diferentes grados de protección (Lugares de Interés Comunitario, Zonas de Espacial Protección de Aves, etc.).

Por otra parte, la escala subregional y supramunicipal resulta interesante y adecuada para testear la metodología propuesta en contextos metropolitanos.

\section{La propuesta metodológica}

La propuesta se fundamenta en la evaluación de cuatro de las dimensiones de la IV, empleando para ello herramientas de análisis espacial propias de los SIG y otras de la ecología del paisaje, junto a métodos participativos, como valoraciones expertas. Para ello se empleará un entorno de trabajo SIG fundamentalmente ráster. La propuesta se articula en tres fases que se describen en los siguientes apartados: (1) Evaluación y cartografiado de las dimensiones de la IV; (2) Integración de las mismas mediante evaluación multicriterio y generación de un índice global de IV; (3) Selección de áreas potenciales para su integración en la IV.

\subsection{Evaluación y cartografía de las dimensiones de la IV}

En esta primera fase se realiza una evaluación y cartografiado de cuatro de las dimensiones que confluyen en el concepto de IV. Dichas dimensiones quedan recogidas en la figura 2 y se detallan a continuación.

\section{Contribución a los servicios ecosistémicos (SE)}

La multifuncionalidad, entendida como la capacidad del territorio para acoger diferentes actividades, servicios y funciones (ecológicas, sociales, económicas, etc.) (DGMA, 2011), es uno de los principios o dimensiones centrales en la definición y planificación de la IV. Para evaluar dicha multifuncionalidad se propone adoptar el enfoque de valoración y cartografía de la contribución de diferentes elementos territoriales al mantenimiento de los SE, asumiendo que cuanto mayor sea dicha valoración, mayor será el interés de esos elementos para formar parte de la IV. La evaluación de dicha contribución se convierte así en el elemento central de esta dimensión. 
Figura 2. Proceso metodológico general de la propuesta para definir la IV

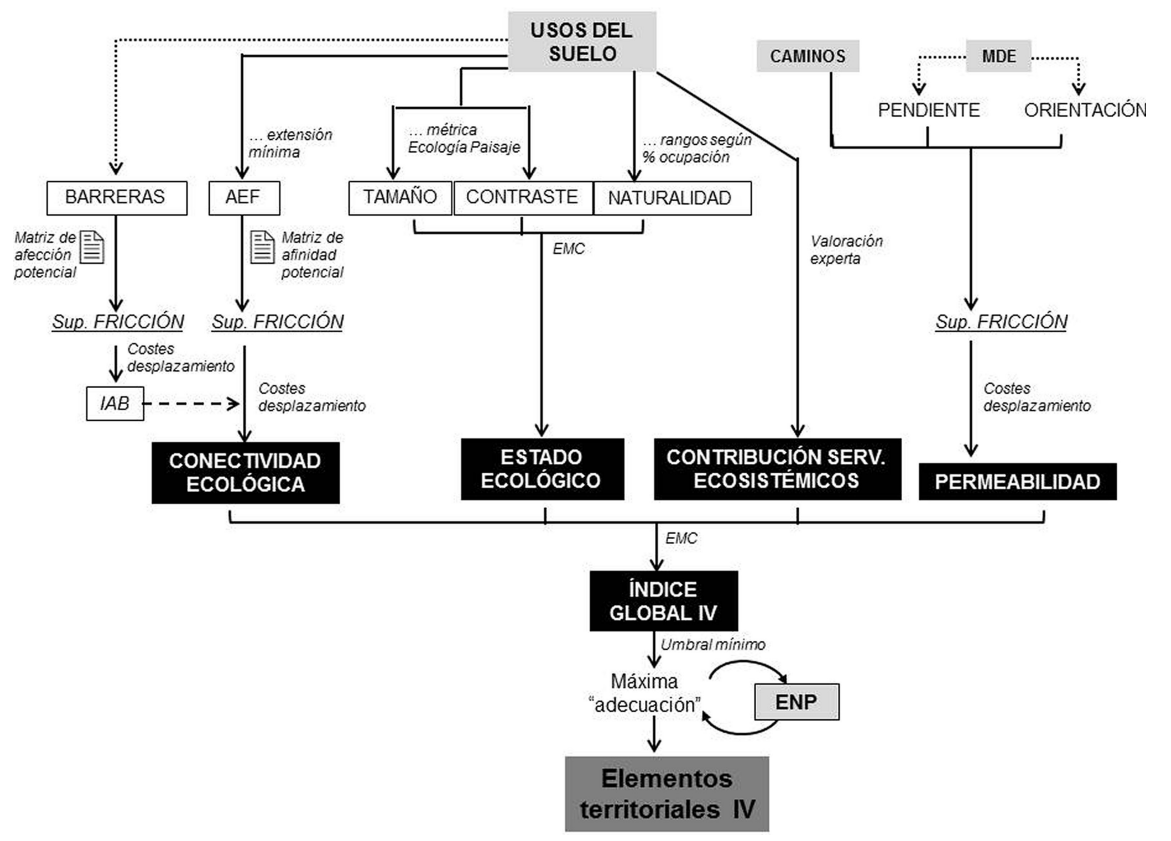

Fuente: elaboración propia.

En los trabajos existentes en la materia es habitual reconocer la validez de las valoraciones expertas para determinar la contribución de diferentes elementos territoriales a la provisión de dichos servicios (Hausen y Pauleit, 2014). Para ello se suele recurrir a diversas técnicas participativas, talleres, sesiones de discusión, etc. en los que implicar a los diferentes actores del proceso de planificación (Hausen y Pauleit, 2014; Kopperoinen et al., 2014), así como al uso de paneles de expertos que, aunque pueden ser más limitados que otras técnicas participativas, suelen resultar igualmente operativas.

La evaluación y cartografía de la contribución a SE se realiza en varias fases:

1) Identificación y selección de elementos con contribución a la prestación de SE: aunque la dimensión espacial de los SE puede ser discutida, es habitual adoptar unidades espaciales a las que referirlos. A este respecto, se han tomado como elementos para su valoración una selección de categorías de usos del suelo (Hausen y Pauleit, 2014; Kopperoinen et al., 2014; Liquete et al., 2015, entre otros) extraídas de SIOSE, teniendo en cuenta algunas relaciones orientativas de elementos a incluir en las IV: CE (2010); EEA (2011); DGMA (2011) o la recogida en la guía Technical Information on Green Infrastructure for Europe (EC, 2013), entre otros.

2) Selección de SE a evaluar: la variedad de SE es muy amplia (Kopperoinen et al., 2014), existiendo varias clasificaciones propuestas desde diferentes orga- 
nismos. Liquete et al. (2015) recomiendan utilizar la de TEEB-The Economics of Ecosystems and Biodiversity (<http://www.teebweb.org/resources/ ecosystem-services/>) o la de CICES-Common International Classification of Ecosystem Services (<http://cices.eu/>). Por su parte, la Agencia Europea de Medio Ambiente ofrece una clasificación en la que los servicios se agrupan en cuatro categorías generales (de hábitat, relacionadas con la conservación de la biodiversidad; de regulación de procesos ecológicos que contribuyan a la mitigación y adaptación al cambio climático; de abastecimiento, de alimentos y materias primas, y gestión del ciclo del agua; y, por último, los servicios culturales que contribuyen al bienestar y salud de la población o a su enriquecimiento cultural), que, a su vez, pueden desglosarse en otras de mayor detalle (EEA, 2011).

La propuesta emplea un número de servicios a evaluar no demasiado extenso (quedándose en el segundo nivel de desagregación de esta última clasificación) para incentivar y facilitar la participación de los expertos del panel.

3) Valoración cualitativa mediante panel de expertos y cartografía: seleccionados elementos y servicios, un panel, concretado en forma de encuesta sencilla dirigida a una veintena de expertos (de diferentes disciplinas y ámbitos relacionados con la planificación y gestión del territorio), valoró cualitativamente (entre 1-ninguna a 4-mucha) la contribución de cada uso del suelo a cada servicio. Para obtener la valoración cuantitativa final de la contribución potencial de cada uso del suelo se optó por aplicar el estadístico de moda a las valoraciones obtenidas del panel de expertos. De manera alternativa podrían utilizarse operaciones aritméticas con las que agregar las valoraciones individuales y así obtener un valor global de contribución al conjunto de servicios.

Finalmente, a partir de la valoración obtenida para cada elemento, se realiza la cartografía, asignando a cada uno de los usos su correspondiente valor de contribución a los SE.

\section{Conectividad ecológica}

La conectividad, al igual que la multifuncionalidad, es otra de las dimensiones centrales de la IV, ya que los espacios protegidos no son suficientes por sí mismos y aislados para mantener la biodiversidad y asegurar el adecuado funcionamiento, la continuidad y coherencia de la IV como sistema (Gurrutxaga et al., 2015). Es por ello que la conectividad de un paisaje o territorio contribuye a la conservación de espacios naturales, al incremento de su resiliencia y, en definitiva, a asegurar y preservar la biodiversidad y también la provisión de bienes y servicios con los que satisfacer necesidades de la población (Sbara et al., 2007; CE, 2010; DGMA, 2011; Remolina, 2011; CE, 2013).

Sin embargo, la conectividad es un concepto difícil de hacer operativo, lo que ha llevado a la aparición de numerosas aproximaciones para su cuantificación y cartografía (Pla et al., 2007). Aunque actualmente existen diferentes 
programas y aplicaciones, o módulos específicos dentro de ellos, destinados al análisis de la conectividad (Guidos, Fragstat, ArcGIS, etc.), abundan también los estudios que recurren a los análisis de costes incluidos en los SIG para modelar la denominada conectividad funcional, la definida en términos de las necesidades requeridas por especies determinadas (Adriaensen et al., 2003; Cushman et al., 2008; Theobald et al., 2006; Gonzales y Gergel, 2007), a partir de superficies ráster de fricción. Como resultado de estos trabajos, se deriva, en un entorno ráster, un índice de conectividad ecológica de cada punto (píxel) del territorio. Los trabajos de Marull y Mallarach, recogidos en varias publicaciones (Marull y Mallarach, 2002, 2005; Mallarach, 2004, entre otras), constituyen una valiosa aportación en esta línea, presentando una propuesta metodológica sólida y fácilmente replicable y que será la adoptada en esta propuesta.

Definida como una metodología "de tipo paramétrico», el cálculo del índice de conectividad ecológica final pasa por tres fases consecutivas:

1) Áreas Ecológicas Funcionales (AEF): en un primer momento, el objetivo es identificar las áreas mínimas para cada tipo de cubierta donde se pueden desarrollar comunidades animales representativas o de interés y que, "por sus características intrinsecas y contextuales, deben preservarse y relacionarse mediante una red de conectores que aseguren los flujos» (Marull y Mallarach, 2002: 4). A partir de la cobertura de usos del suelo, se identifican y agrupan según afinidad aquellos usos que mejor definirían las AEF (bosques, cultivos en secano, matorrales, etc.), seleccionando parcelas con superficie mínima necesaria para constituirlas (figura 3).

2) Índice de Afección de Barreras (IAB): su cálculo se aborda como un análisis de costes de desplazamiento. En primer lugar, el procedimiento pasa necesariamente por identificar aquellos usos del suelo presentes en el territorio que pueden actuar como barreras a la conectividad ecológica (zonas urbanizadas, infraestructuras viarias, etc.). Por otra parte, la superficie de fricción se genera a partir de una «matriz de afección potencial» en la que se valoran los costes de atravesar el territorio en función de la presencia y tipología de barreras y del uso del suelo existente: vegetación agrícola, vegetación forestal, zonas neutras y barreras. Finalmente, el índice resulta de la superposición aritmética de efectos barrera individuales, en los que también se ha considerado la relación inversa entre estos y la distancia.

3) Índice de Conectividad Ecológica (ICE): como en el índice anterior, en la propuesta de estos autores se recurre al análisis de coste de desplazamiento. Aquí, el origen se sitúa en cada una de las AEF definidas previamente y la superficie de fricción se deriva de una «matriz de afinidad potencial» en la que, a partir del conocimiento experto, se valora el coste de llegar a una unidad a otra del mismo tipo atravesando zonas con distinto grado de afinidad.

\section{Accesibilidad/permeabilidad a la población}

Favorecer la permeabilidad, no solo biológica, de la IV con el objetivo final de alcanzar el bienestar y una mayor calidad de vida de la población, debe ser otra 
Figura 3. Proceso metodológico para generar AEF

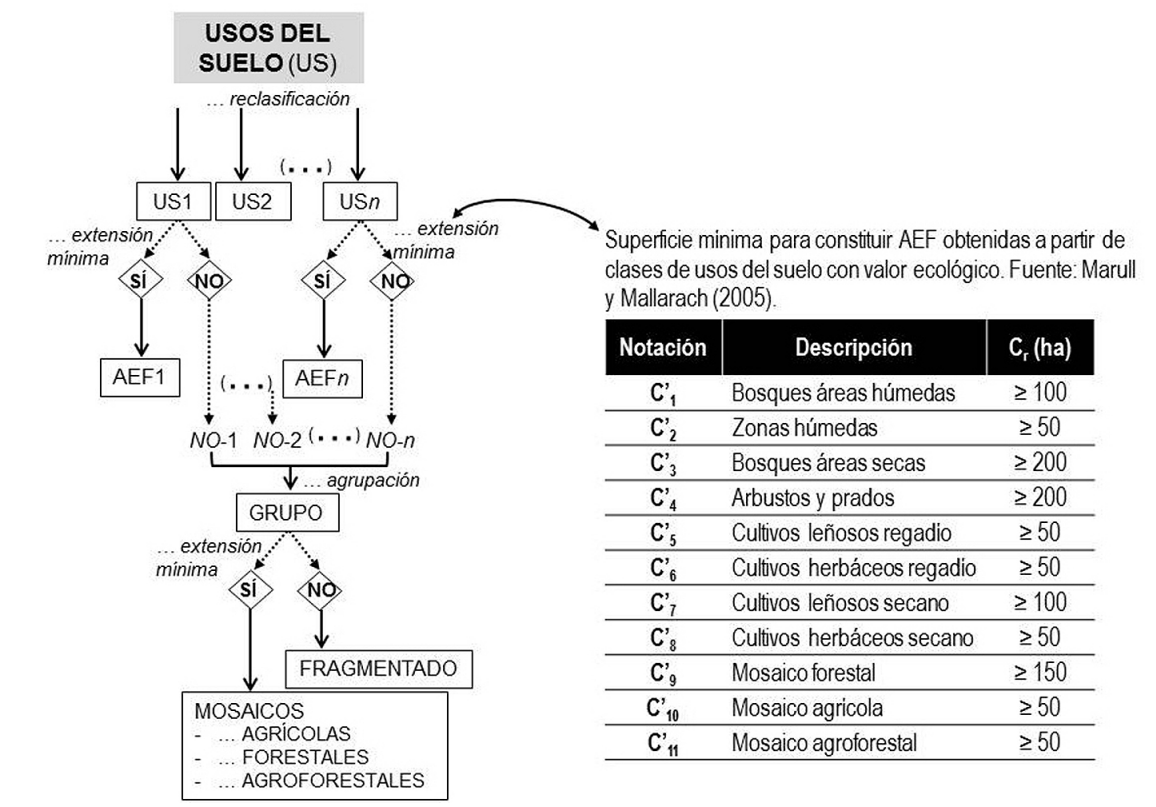

Fuente: elaboración propia.

de las dimensiones presentes a la hora de planificar estas infraestructuras, facilitando así el acceso de la población al conjunto de bienes y servicios que sus elementos pueden ofrecer. Más aún si se entiende que los diferentes elementos de la infraestructura tienen vocación de equipamiento (Fariña, 2012), por lo que deberían aportar espacios para el esparcimiento de los ciudadanos, para la socialización o la realización de diferentes actividades en contacto con la naturaleza.

La valoración de esta dimensión tiene como objetivo medir el grado de permeabilidad del territorio a la población, para priorizar aquellos espacios a los que el acceso, en desplazamientos a pie y para disfrutar o desarrollar diferentes tipos de actividades, sea más favorable. Para ello se propone recurrir de nuevo a los análisis de costes de desplazamiento incluidos en los SIG; en este caso, desplazamientos a través de la red viaria, preferentemente de carácter peatonal y con origen en los núcleos urbanos.

Existe consenso en considerar a la topografía, en general, y a la pendiente, en particular, como factor determinante de las posibilidades para desplazarse sobre un terreno (López Romero, 2005), más en desplazamientos a pie. Al generar las superficies de fricción necesarias para calcular los costes de desplazamiento se asume, de forma general, que estos se ven favorecidos (exigen menor esfuerzo energético) en terrenos llanos, de escasa pendiente.

En este punto de la propuesta se ha optado por abordar los análisis de costes a partir de superficies anisotrópicas. Frente a las superficies isotrópicas, aquellas 
modelan de forma más realista el proceso, al considerar que los costes unitarios varían irregularmente dependiendo no solo de la línea de máxima pendiente, sino también de la dirección de la misma, cuesta arriba o cuesta abajo. Su implementación toma en cuenta, además de las pendientes del área de estudio, las orientaciones del terreno, añadiendo una cierta complejidad en la interpretación de los resultados (Romo y Torres, 2011; Wood y Schmidtlein, 2012).

Para generar los costes a través de la red se recurre a la cartografía BTN25 y al MDT25, mediante un análisis de proximidad al viario y la asignación a cada unidad espacial obtenida del valor de coste del tramo del viario más próximo. El resultado conseguido es una superficie continua de costes para toda el área de estudio que constituye otra de las dimensiones de la IV.

\section{Estado ecológico}

Esta dimensión tiene como objetivo evaluar el estado de los usos y coberturas del suelo que integran el paisaje, en función de su composición y configuración (McGarigal, 2015), aportando una aproximación sencilla al estado ecológico. Para ello se han seleccionado algunas características de la configuración espacial de los usos del suelo, como el grado de naturalidad, el tamaño de las teselas de dichos usos o el contraste entre usos adyacentes. Así, una zona o sector del paisaje con un predominio de usos artificiales presentaría un peor estado ecológico, frente a otro donde predominen los usos naturales. Por otra parte, aquellas zonas donde los usos estén muy fragmentados, porque los tamaños de sus teselas son muy pequeños, presentarán peor estado que aquellas donde los usos no estén altamente fragmentados. A continuación, se describe cómo se han determinado estas características en la presente propuesta.

1) Tamaño de las teselas: se generó rasterizando la capa vectorial de usos y coberturas del suelo categorizada, asignando como valor del ráster resultante el tamaño de cada una de dichas teselas.

2) Naturalidad: aunque resulta difícil evaluar en la práctica el concepto de naturalidad (García Leyton, 2004), y que clasificar usos/coberturas del suelo en función de su naturalidad puede ser bastante cuestionable, su realización no tiene por qué ser arbitraria (Machado et al., 2004). Así, es posible encontrar multitud de índices que intentan materializar este concepto (Bosque et al., 1997; García Leyton, 2004; Estévez, 2012; Oñate et al., 2014, entre otros). En la presente propuesta se ha considerado la proporción de extensión de cubiertas de tipo natural en relación con la superficie total del ámbito de estudio (figura 4), asignando el nivel máximo de 4 a aquellos espacios con elevados porcentajes de extensión de cubiertas naturales (arbolado en general, láminas de agua, etc.), frente a un mínimo de 1 para los espacios predominantemente artificiales (alto porcentaje de extensión ocupada por edificaciones, viales, etc.).

A este respecto, la información que ofrece SIOSE sin categorizar permite, mediante búsqueda selectiva por atributo en un SIG, generar diferentes capas (una por cada tipo de uso/cubierta y por cada nivel de naturalidad) a las que 
Figura 4. Proceso metodológico para generar grados de naturalidad según porcentaje de cobertura de usos del suelo

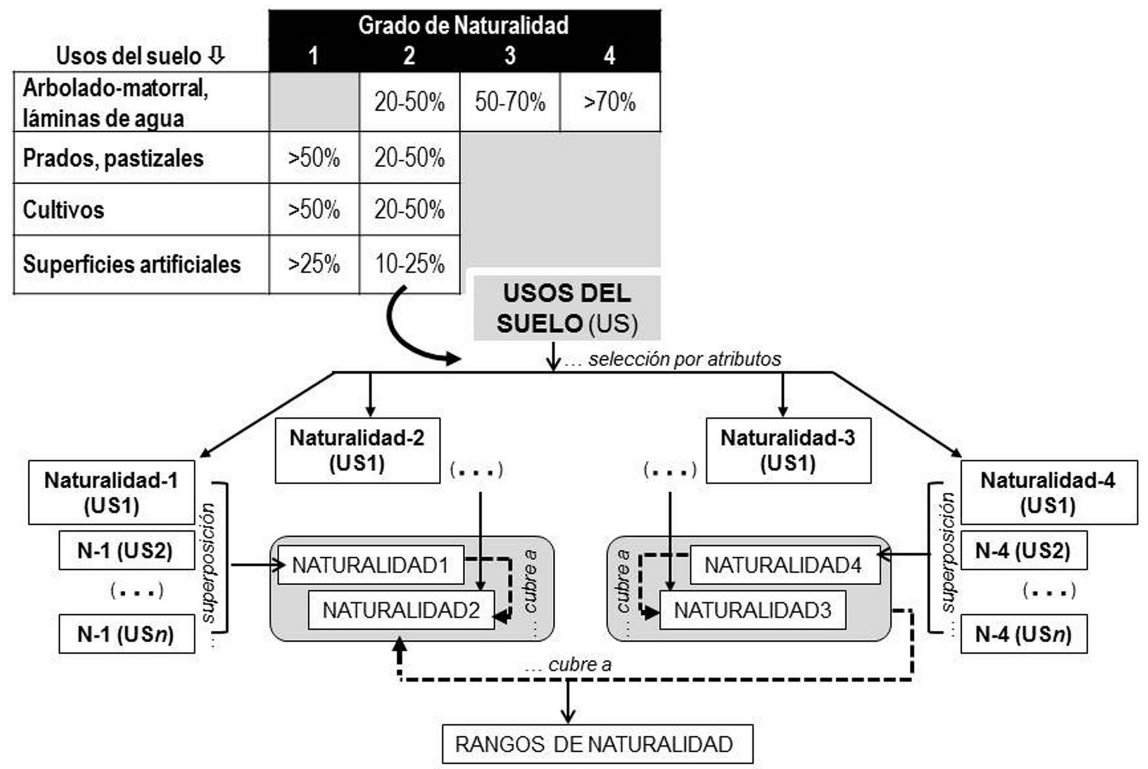

Fuente: elaboración propia.

asignar el correspondiente valor de naturalidad. De esta forma, una misma tesela obtiene diferentes valores de naturalidad (en función del número de cubiertas presentes en ella), por lo que dichos valores se combinan asignando el mayor o el menor de entre los grados de naturalidad obtenidos para cada tesela.

3) Contraste: entendido como la magnitud de la diferencia entre las características ecológicas de un tipo de uso/cobertura del suelo y los adyacentes (McGarigal, 2015), el contraste presenta múltiples implicaciones ecológicas. Una de ellas está relacionada con el mayor grado de aislamiento de aquellas teselas rodeadas de otras con las que comparten pocas características (es decir, zonas de elevado contraste), que podría asociarse con una mayor presión o impacto y, por tanto, con un peor estado ecológico. De esta forma, aquellos píxeles con mayor valor de contraste tendrían menor interés para integrarse en la IV. La estimación del contraste puede realizarse mediante métricas como Edge contrast (ECON_MN) y el programa Fragstats (McGarigal, 2015). Sin embargo, para calcular esta métrica obteniendo un valor para todos los píxeles de un área de estudio, es necesario recurrir a su cálculo mediante ventana móvil (Díaz-Varela et al., 2009). Este proceso consiste en el cálculo mediante un kernel, que obtiene un valor de la métrica para cada píxel, en función de los usos/coberturas del suelo presentes en el kernel de cálculo. La determinación del tamaño del kernel o ventana más adecuado se convierte en un elemento fundamental en esta aproximación. 
Figura 5. a) Cambio en disimilaridad para métrica calculada con ventana móvil en función del tamaño de ventana (tamaño elegido, $1.200 \mathrm{~m}$ ). b) Contraste resultante de métrica ECON_MN (Corredor del Henares)
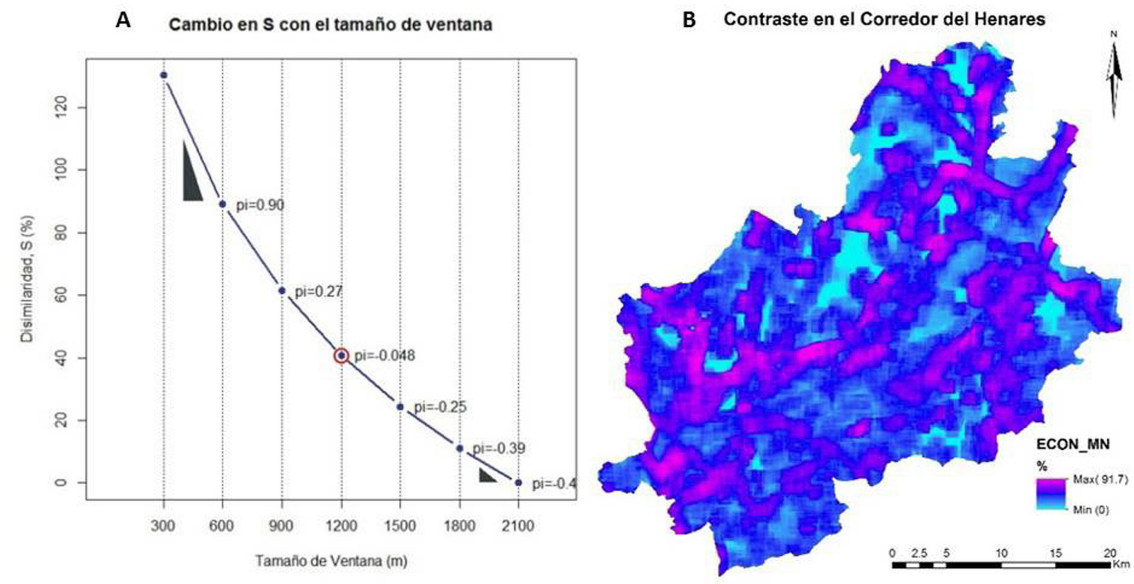

Fuente: elaboración propia.

De acuerdo con diversos trabajos, el tamaño adecuado sería aquel a partir del cual los incrementos de tamaño de la ventana no suponen grandes incrementos en el valor de la métrica, lo que coincidiría con el comienzo del dominio de la mesoescala del paisaje (Díaz-Varela et al., 2009). Para ello, y tras obtener los resultados de la métrica con diferentes tamaños de ventana, aquel valor $(\mathrm{Pi})$ a partir del cual los cambios en porcentaje de los valores de la métrica $(\mathrm{Si})$ sean menores que los cambios en porcentaje del tamaño de ventana (Wi) permite seleccionar el tamaño de ventana adecuado, lo que es equivalente a que (figura 5a):

$$
P i=\frac{\Delta S i}{\Delta W i}-1<0
$$

Por otra parte, la métrica ECON_MN requiere de la definición de una matriz de contraste entre categorías de usos/coberturas que especifique, en un rango de 0 a 1, el nivel de contraste entre cada par de usos (McGarigal, 2015). La aproximación propuesta consiste en establecer 5 niveles de contraste entre los usos, partiendo de nulo (0 valor de contraste), 0,25 (valor bajo), 0,5 (valor medio), 0,75 (valor alto) y llegando hasta 1 (máximo) que permitiría el cálculo de la métrica (figura $5 b)$.

A continuación, los tres componentes del estado ecológico se combinan mediante el uso de métodos compensatorios de EMC (Gómez Delgado y Barredo Cano, 2005), la sumatoria lineal ponderada. 
Figura 6. Cartografía de las dimensiones de una IV para el Corredor del Henares (MadridGuadalajara): a) Contribución global a prestación de Servicios Ecosistémicos; $b$ ) Índice de Conectividad Ecológica; c) Accesibilidad al territorio (costes anisotrópicos a través de la red);

d) Estado ecológico de usos y coberturas del suelo

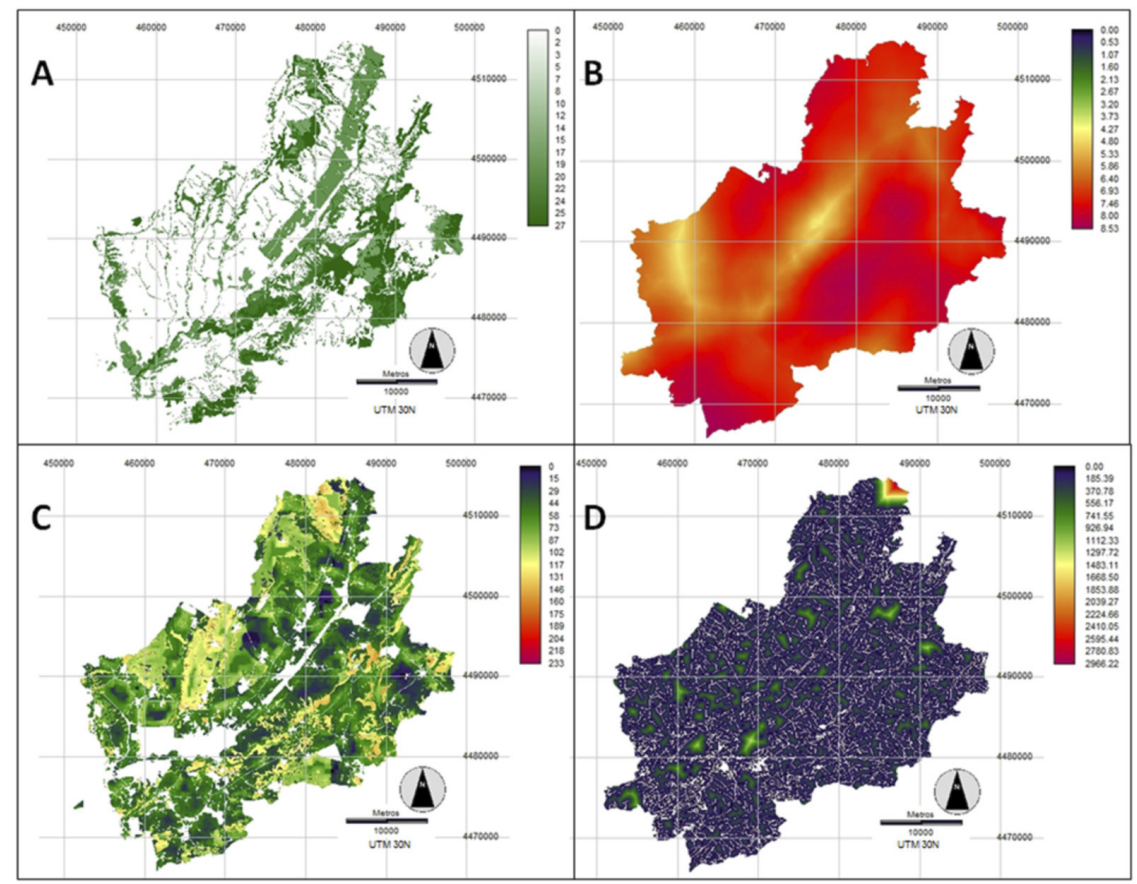

Fuente: elaboración propia.

Finalmente, en la figura 6 se muestran los resultados de la aplicación de la propuesta metodológica a la cartografía en el Corredor del Henares de las cuatro dimensiones de IV consideradas.

\subsection{Integración mediante EMC}

La segunda fase de la propuesta tiene como objetivo integrar las dimensiones de la IV evaluadas y cartografiadas para obtener una evaluación de la potencialidad de cada punto del territorio para integrarse en la IV. En ese sentido, existen varias posibilidades, desde la creación de un índice sintético utilizando algún método matemático-estadístico hasta la aplicación de técnicas de EMC (y multiobjetivo), ampliamente utilizadas en el ámbito de la planificación territorial en los últimos años. Estas últimas son las elegidas en esta propuesta dado que ofrecen la suficiente flexibilidad para que el resultado final sea consensuado por todos los agentes implicados en el proceso de planificación, pues ofrecen la oportunidad de obtener un análisis equilibrado de todas las facetas del problema (Nijkamp y Van Delft, 1977). 
El primer paso para integrar las cuatro dimensiones mediante evaluación multicriterio es comprobar que no se encuentran correlacionadas. Si fuera así, alguna de ellas debería ser eliminada o, en el caso de que se fuese a proceder a establecer algún tipo de ponderación, debería repartirse el peso entre las dimensiones correlacionadas para no sesgar los resultados (Gómez Delgado y Barredo Cano, 2005). En este caso, la aplicación del análisis de regresión a las dimensiones de la IV consideradas ofreció valores de correlación bajos, con coeficientes de determinación $r$ inferiores, en todo caso, a 0,5 .

A continuación, se procede a la normalización de las cuatro dimensiones ya que es necesario que la EMC se realice con medidas variables sobre escalas comparables en tipo, rango de extensión, unidad de medida, eventual posición del cero, dispersión, etc. (Barba-Romero y Pomerol, 1997). La opción empleada fue el ajuste lineal, aunque podrían emplearse otros métodos, por ejemplo, dividiendo cada valor por el máximo o restando a cada valor el mínimo y dividiéndolo por la diferencia entre el máximo valor y el mínimo (Voogd, 1983) o incluso emplear métodos más flexibles, como los basados en funciones de pertenencia a un conjunto borroso (Malczewski, 1999).

A continuación, se combinan las cuatro dimensiones normalizadas mediante una de las múltiples técnicas de EMC existentes (figura 7). En este caso se ha optado por utilizar una de las más sencillas en su formulación matemática, como es la técnica compensatoria de la sumatoria lineal ponderada, para que los resultados sean así también más entendibles y puedan ser fácilmente analizados por los agentes implicados en cualquier proceso de este tipo.

Para realizar la ponderación de los distintos factores en la sumatoria lineal, se han asignado pesos iguales a las diferentes dimensiones (factores) de la IV

Figura 7. Clasificación de técnicas EMC

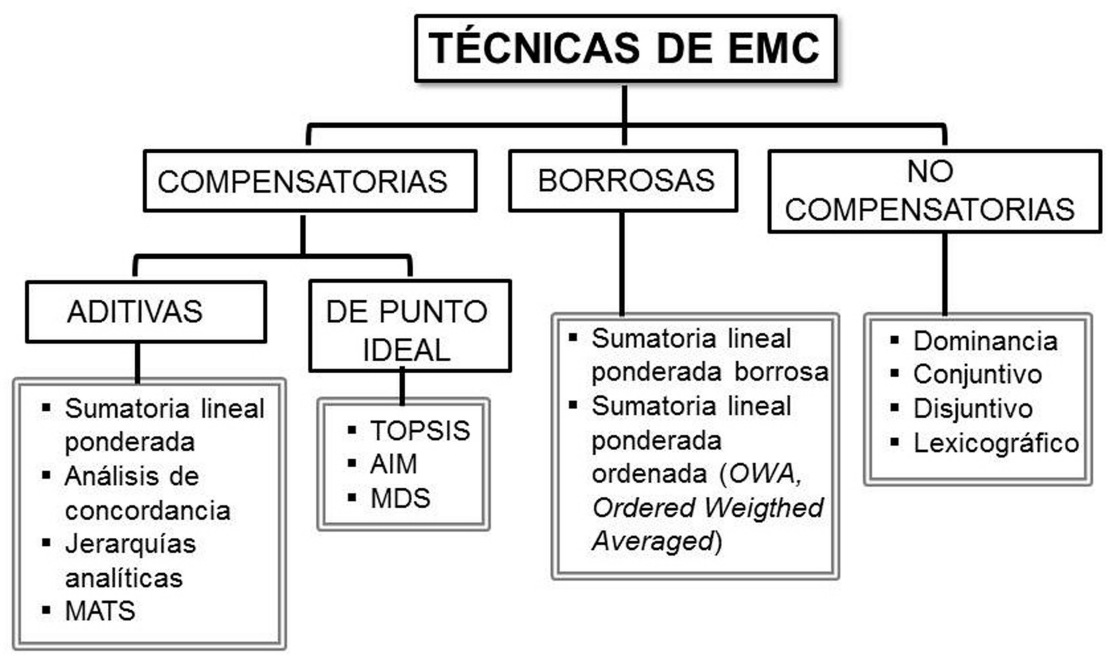

Fuente: modificado de Jankowski (1995). 
Figura 8. a) Cartografía de nivel de adecuación del territorio para la IV en el Corredor del Henares (Madrid-Guadalajara); b) Histograma de frecuencias y medidas estadísticas para determinar umbrales mínimos de adecuación; c) ZPIV tras la aplicación del cuartil 1 para establecer el umbral mínimo de adecuación para la IV

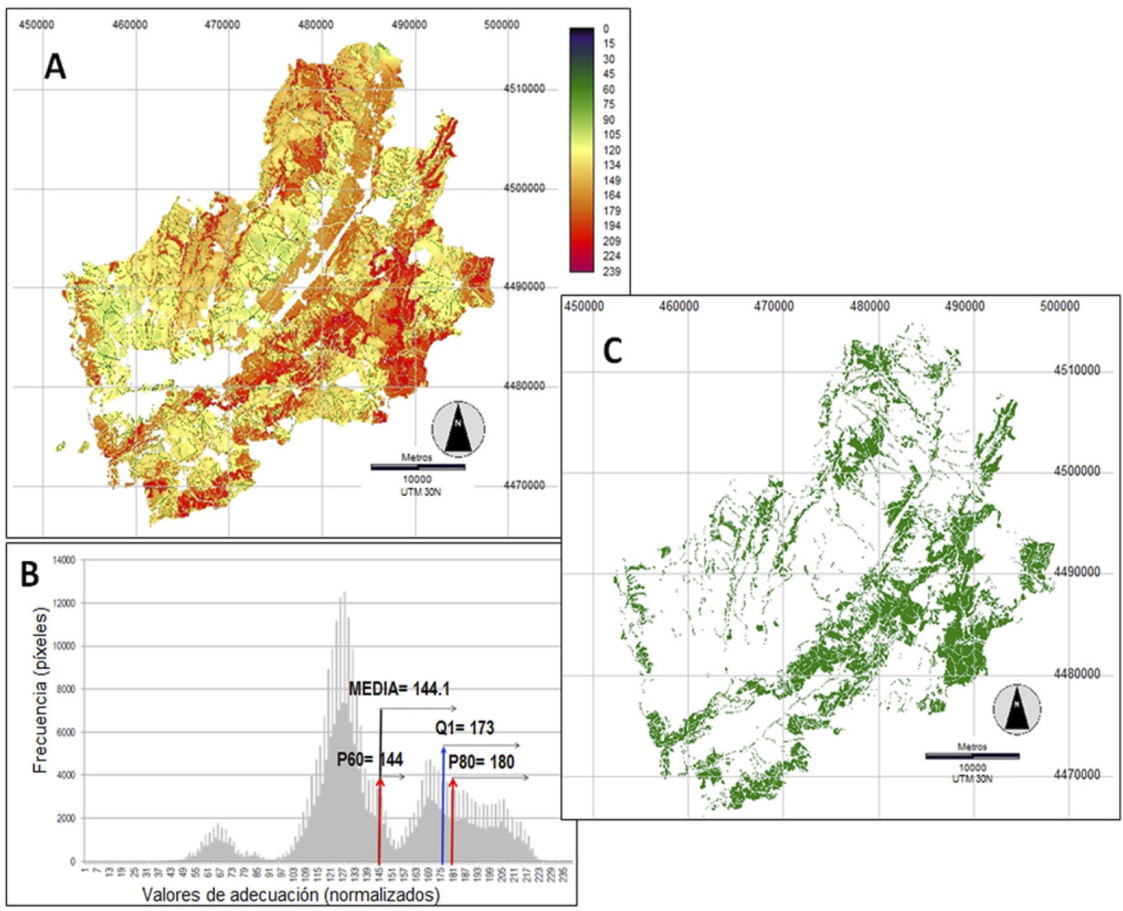

Fuente: elaboración propia.

que se integrarán en la regla de decisión aplicada. No obstante, sería posible igualmente utilizar alguno de los variados y abundantes métodos existentes, como la matriz de comparación por pares de Saaty, el método de la entropía, el método Delphi basado en consultas a paneles de expertos, etc. (Barba-Romero y Pomerol, 1997; Malczewski, 1999).

\subsection{Selección de áreas potenciales para su integración en la IV}

Finalmente, el resultado de la EMC es un mapa de adecuación con un valor individual para cada píxel que resume la valoración de las cuatro dimensiones de la IV (figura 8a). Para poder identificar zonas o píxeles con potencial para integrarse en la IV (ZPIV) se recurre a analizar la distribución de frecuencias de adecuación resultante para, así, establecer el conjunto de píxeles a retener, empleando un valor umbral (figura 8b). Ante la falta de normalidad de los datos, se ha utilizado una medida de posición con la que mantener los valores comprendidos dentro del primer cuartil (figura 8c), obteniendo finalmente el mapa de ZPIV. 
Figura 9. Cartografía de la IV en el Corredor del Henares (Madrid-Guadalajara)

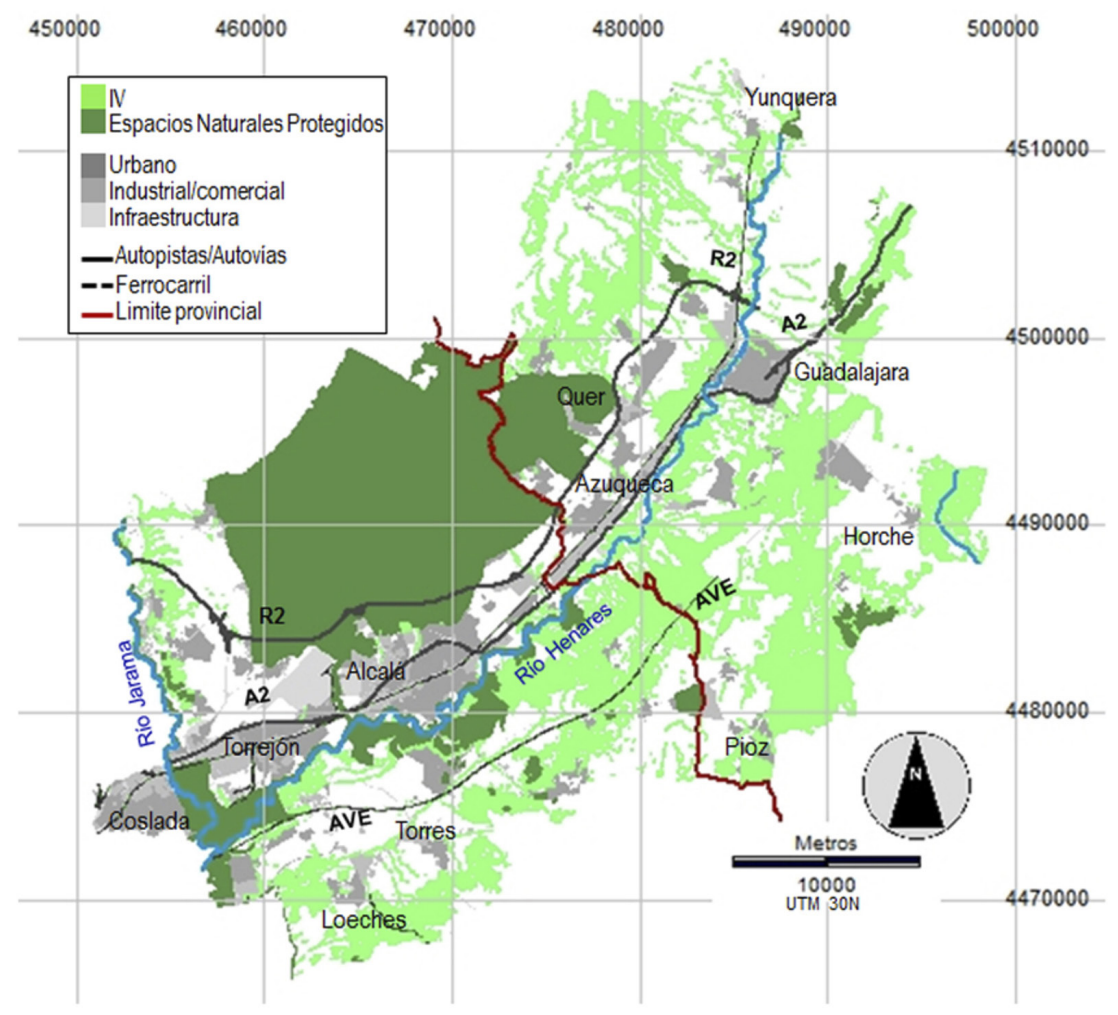

Fuente: elaboración propia.

A partir de este resultado es posible ofrecer una cartografía final de las zonas con potencial para incorporarse a la IV del área de estudio (figura 9). Si bien, un último aspecto a tener en cuenta para completar dicha propuesta sería la necesaria multiescalaridad de la misma, que implicaría desarrollarla a escalas de mayor detalle, incorporando a la infraestructura elementos territoriales difícilmente identificables a las escalas de la información de partida presentada. De esta forma se podrían, por ejemplo, articular conexiones entre los núcleos de población y la IV, favoreciendo de este modo la dimensión de permeabilidad y acceso de la población a la misma.

\section{Discusión y conclusiones}

Se ha presentado una propuesta metodológica para el diseño espacialmente explícito de una IV empleando herramientas propias de análisis espacial, evaluación multicriterio y ecología del paisaje, testeándose en el Corredor del 
Henares (Madrid-Guadalajara) y a una escala intermedia (subregional). Pretende ser un procedimiento accesible, replicable y sencillo, basado en métodos suficientemente aceptados y contrastados en diversas disciplinas y con un marcado carácter espacial o territorial; por ejemplo, los trabajos sobre conectividad ecológica de Marull y Mallarach (2005), los análisis de estructura del paisaje basados en métricas con ventana móvil (Díaz-Varela et al., 2009); la aplicación de técnicas participativas para valorar la contribución a la prestación de SE (Kopperoinen et al., 2014), etc.

A pesar de sus limitaciones, que se discuten más adelante, la oportunidad y necesidad de propuestas similares resulta innegable por el creciente interés del concepto de IV como enfoque innovador, que puede aportar un excelente instrumento para un planeamiento más sostenible y equilibrado con el que orientar los nuevos desarrollos urbanísticos de los planes territoriales y municipales.

Ante la propuesta de modificación de la Ley de Patrimonio Natural y de la Biodiversidad (aún en trámite de aprobación) que por primera vez recoge una estrategia estatal de IV, la oportunidad es mayor y más si cabe al constatar la evidente ausencia del concepto en los planes territoriales españoles (Rodríguez y Aguilera, 2016) y, por tanto, la falta de herramientas que permitan su delimitación, a pesar de la existencia de conceptos similares (malla verde, espacios libres, etc.).

De esta forma, la propuesta incide en la espacialización de estas infraestructuras partiendo de un análisis espacial ráster, superando así el uso de elementos gráficos (flechas, esquemas, etc.), muy frecuente en la representación de la articulación de elementos de valor ambiental en los planes territoriales (Rodríguez y Aguilera, 2016).

En este sentido, se puede comprobar cómo los SIG, al igual que en otros ámbitos, se revelan como una potente herramienta para facilitar la integración y espacialización de las dimensiones y elementos considerados en el diseño de las IV; para integrar fuentes de información diversas y manejar diferentes modelos de datos; para aplicar métodos y herramientas de diversos ámbitos disciplinares, etc., constituyendo así una muestra más de la aplicabilidad de estos sistemas a la resolución de problemas territoriales.

No obstante, esta propuesta tiene vocación de continuar desarrollándose, por ejemplo, mediante la concreción de los elementos territoriales a incorporar en ella, ya que, como se ha podido comprobar, las ZPIV estarían compuestas por grupos de píxeles o incluso algunos píxeles aislados que no se corresponden con "piezas» del territorio. De esta forma, mediante la superposición de elementos territoriales, como usos del suelo, red de caminos, vías pecuarias, red hidrográfica, etc., se podrían identificar los elementos territoriales con un grado de coincidencia elevado con las ZPIV, que se convertirían en los auténticos elementos de la IV.

Igualmente, estos elementos territoriales se deberían integrar con los Espacios Naturales Protegidos (ENP) existentes en el área de estudio (Reservas de la Biosfera, Red Natura2000, Parques Nacionales, Parques Naturales o Regionales, etc.), con objeto de asegurar la conexión con los mismos, ya que 
es incuestionable la importancia de estos como elementos nucleares de toda IV desde donde difundir sus valores y servicios ambientales.

Otra línea de progreso es la consideración de la multiescalaridad en la implementación de la propuesta de IV, que exigiría la concreción a escalas de mayor detalle (más cercanas a la escala de planificación municipal) de los elementos territoriales integrantes, así como, y especialmente, la relación y articulación de los mismos con los núcleos urbanos, con el objetivo de que se garantice el acceso de la población a los elementos de la IV.

Entre las limitaciones que se reconocen estaría la relacionada con la integración de factores mediante técnicas EMC, proceso que mantiene ciertos grados de subjetividad en aspectos como, por ejemplo, la definición de pesos a asignar o para determinar valores umbral que definirán las ZPIV. El conocimiento de la realidad, así como la participación de agentes y profesionales con implicación y conocimiento experto sobre el ámbito en cuestión pueden aportar elementos de juicio al respecto.

En esta línea, otra limitación sería la necesidad de conocer e integrar herramientas y análisis espaciales diversos, que, pese a su demostrado valor e interés en el proceso planificador, continúan restringidas al ámbito académico. Esto puede dificultar el salto hacia la praxis profesional y, por tanto, limitar la aplicabilidad de la propuesta. Resultaría oportuno y deseable avanzar en el empleo de técnicas participativas (workshops, entrevistas, etc.) con agentes implicados en el proceso de planificación, tanto para testear la implementación de la propuesta como para incorporar y adaptar sugerencias, necesidades y requerimientos de todos los actores implicados (Hewitt et al., 2014; Soria-Lara et al., 2016), constituyendo igualmente una línea de desarrollo de la presente propuesta.

También, en relación con los datos de partida, cabe mencionar que la elección de la cartografía SIOSE (en cualquiera de sus versiones temporales) presenta como aspecto positivo el ofrecer información interesante sobre porcentajes de cobertura por tesela, útiles en la valoración de la conectividad o la naturalidad, entre otros. Pero, en contrapartida, para otros análisis (de accesibilidad, de valoración de SE, etc.) exige ser categorizada, asignando usos o coberturas predominantes a todos y cada uno de los polígonos de la base de datos original, en un procedimiento que no siempre resulta obvio y que implica cierta complejidad.

Finalmente, es posible concluir que no hay duda del interés y oportunidad que presentan este tipo de infraestructuras para, como se ha mencionado con anterioridad, dotar al planeamiento de un enfoque innovador e integrador de aspectos ambientales, territoriales, dotacionales, etc. De esta forma será posible contribuir a que la ordenación y planificación adquieran un auténtico carácter estratégico e integrado, algo tan necesario en ámbitos metropolitanos o interregionales en el caso de nuestro país, con el que orientar, reorientar y articular futuros desarrollos, así como con el objetivo de ampliar, mejorar y garantizar la efectiva integración de espacios urbanos, rurales y naturales en el territorio. Por tanto, y aunque se sabe de la necesidad de abordar el diseño de la IV desde 
la multiescalaridad y de la integración con elementos territoriales reales, los autores entienden que la presente propuesta constituye una primera aproximación valiosa que contribuye al desarrollo de metodologías que permitan el diseño y delimitación espacial de los componentes territoriales de la IV desde una perspectiva práctica y desde un marco conceptual sencillo pero riguroso, que pueda ser mejorado y revisado en posteriores trabajos o por otros autores.

\section{Referencias bibliográficas}

Adriaensen, F.; Chardon, J. P.; De Bulst, G.; Swinnen, E.; Villalba, S.; GilincK, H. y MATTHYSEN, E. (2003). «The application of least cost modeling as a functional landscape model». Landscape an Urban Planning, 64, 233-247. <https://doi.org/10.1016/S0169-2046(02)00242-6>

Barba-Romero, S. y Pomerol, J. CH. (1997). Decisiones multicriterio. Fundamentos teóricos y utilización práctica. Alcalá de Henares: Servicio de Publicaciones de la Universidad de Alcalá.

Benedict, M. A. y MCMAHOn, E. T. (2006). Green Infrastructure. Linking Landscapes and Communities. Washington, D.C.: Island Press.

Bosque Sendra, J.; Gómez Delgado, M.; Rodríguez Durán, A.E.; Rodríguez Espinosa, V. M. y Vela GaYo, A. (1997). «Valoración de los aspectos visuales del paisaje mediante la utilización de un SIG». Documents d'Anàlisi Geogràfica, 30, 19-38.

CANTARINO MARTí, I. (2013). «Elaboración y validación de un modelo jerárquico derivado de SIOSE». Revista de Teledetección, 39, 5-21.

CE (2010). Una infraestructura verde. Luxemburgo: Directorate-General for the Environment. European Commission-Oficina de Publicaciones Oficiales de la Unión Europea, 4 pp. <http://ec.europa.eu/environment/pubs/pdf/factsheets/green_infra/ es.pdf> [consulta: julio 2016].

- (2013). Infraestructura verde: mejora del capital natural de Europa. Comunicación de la Comisión al Parlamento Europeo, al Consejo, al Comité Económico y Social Europeo y al Comité de las Regiones (COM (2013) 249 final). Bruselas: Comisión Europea, 12 pp. <http://eur-lex.europa.eu/resource.html?uri=cellar:d41348f2-01d5-4abeb817-4c73e6f1b2df.0008.03/DOC_1\&format=PDF> [consulta: julio 2016].

Corredor Camargo, E. S.; Fonseca Carreño, J. A. y Páez Barón, E. M. (2012). «Los servicios ecosistémicos de regulación: tendencias e impacto en el bienestar humano». Revista de Investigación Agraria y Ambiental (RIAA), 3 (1), 77-83.

Cushman, S. A.; McKelveY, K. S. y SCHWARTZ, M. K. (2008). «Use of empirically derived source-destination models to map regional conservation corridors». Conservation Biology, 23, 368-376.

$<$ https://doi.org/10.1111/j.1523-1739.2008.01111.x>

CVOPVT (2011). Plan de Infraestructura Verde y Paisaje de la Comunitat Valenciana (Pendiente de aprobación). Conselleria de Vivienda, Obras Públicas y Vertebración del Territorio. Generalitat Valenciana. Disponible en Cátedra de Municipios Sostenibles, Universitat Politècnica de València. <http://www.upv.es/contenidos/ CAMUNISO/info/778623normalc.html> [consulta: julio 2016].

DGMA (2011). «Infraestructuras verdes, infraestructuras con futuro». Revista Murcia Enclave Natural (digital), 27 (2。 2011, año 9), 32-39. <http://www.murciaenclaveambiental.es/ftp/flash/27/index.htm> [consulta: abril 2013]. 
Díaz Varela, E.; Roces-Díaz, V. y Álvarez-Álvarez, P. (2016). «Detection of landscape heterogeneity at multiple scales: use of Quadratic Entropy Index». Landscape and Urban Planning, 153, 149-159. <https://doi.org/10.1016/j.landurbplan.2016.05.004>

Díaz-Varela, E.; Álvarez-López, C. y Marey-Pérez, M. P. (2009). «Multiscale delineation of landscape planning units based on spatial variation of land-use patterns in Galicia, NW Spain». Landscape Ecological Engineering, 5, 1-10. <https://doi.org/10.1007/s11355-008-0053-4>

EC (2013). Technical information on Green Infrastructure (GI). Commission Staff Working Document SWD (2013) 155 final. Accompanying the document communication from the Commission to the European Parliament, the Council, the European Economic and Social Committee and the Committee of the Regions "Green Infrastructure (GI)-Enhancing Europe's Natural Capital \{COM(2013) 249 final\}». Bruselas: European Commission, 24 pp. <http://ec.europa.eu/environment/nature/ecosystems/docs/green_infrastructures/1_ EN_autre_document_travail_service_part1_v2.pdf> [consulta: julio 2016].

EEA (2007). CLC2006 technical guidelines (Technical report No 17/2007). Copenhagen: European Environment Agency (EEA)-Publications Office of European Union, 66 pp. <https://doi.org/10.2800/12134>

- (2011). Green infrastructure and territorial cohesion. The concept of green infrastructure and its integration into policies using monitoring systems (EEA Technical Report No 18/2011). Copenhagen: European Environment Agency (EEA)-Publications Office of European Union, $138 \mathrm{pp}$. $<$ https://doi.org/10.2800/88266>

Estévez GonzÁlEZ, V. (2012). Calidad y fragilidad visual del paisaje. MCE, fuzzy logic y GIS (trabajo de fin de máster de Tecnologías de la Información Geográfica). Universidad Complutense de Madrid, Facultad de Geografía e Historia. <http:// eprints.ucm.es/16016/> [consulta: abril 2016].

FARIÑA TOJO, J. (2012). «Infraestructura verde urbana», Blog de José Fariña. Urbanismo, territorio, paisaje y sostenibilidad. <https://elblogdefarina.blogspot.com. es/2012/06/infraestructura-verde-urbana.html> [consulta: junio 2016].

Fisher, B.; Turner, R. K. y Morling, P. (2009). «Defining and classifying ecosystem services for decision making». Ecological Economics, 68, 643-653. <https://doi.org/10.1016/j.ecolecon.2008.09.014>

García Leyton, L. A. (2004). Aplicación del análisis multicriterio en la evaluación de impactos ambientales (tesis doctoral). Universitat Politècnica de Catalunya, Departament de Projectes d'Enginyeria. <http://hdl.handle.net/10803/6830> [consulta: abril 2016].

Gómez Delgado, M. y Barredo Cano, J. I. (2005). Sistemas de Información Geográfica y evaluación multicriterio en la ordenación del territorio. Madrid: RA-MA Ed., 304 pp.

Gonzales, E. K. y Vergel, S. E. (2007). «Testing assumptions of cost surface analysis - a tool for invasive species management». Landscape Ecology, 22, 1155-1168. <https://doi.org/10.1007/s10980-007-9106-6>

Gurrutxaga, M.; Marull, J.; Domene, E. y Urrea, J. (2015). «Assessing the Integration of Landscape Connectivity into Comprehensive Spatial Planning in Spain». Landscape Research, 40 (7), 817-833. <https://doi.org/10.1080/01426397.2015.1031096> 
Hansen, R. y Pauleit, S. (2014). «From Multifunctionality to Multiple Ecosystem Service? A Conceptual Framework for Multifunctionality in Green Infrastructure Planning for Urban Areas». AMBIO, 43 (4), 351-529. <https://doi.org/10.1007/s13280-014-0510-2>

HewitT, R.; VAN DELDEN, H. y Escobar, F. (2014). «Participatory land use modelling, pathways to an integrated approach». Environmental Modelling \& Software, 52, 49-165. <https://doi.org/10.1016/j.envsoft.2013.10.019>

IGN (2015). Sistema de Información de Ocupación del Suelo en España-SIOSE2011. Documento Técnico. Instituto Geográfico Nacional-Centro Nacional de Información Geográfica. <http://www.siose.es/SIOSEtheme-theme/documentos/pdf/ Doc_tec_SIOSE2011_v1.1.pdf> [consulta: julio 2016].

- (2016a). Base Topográfica Nacional 1.25.000. Instituto Geográfico NacionalCentro Nacional de Información Geográfica. <https:/www.ign.es/ign/layoutIn/ actividadesBDGbtn25.do> [consulta: julio 2016].

- (2016b). Modelo Digital de Elevaciones MDT25. Instituto Geográfico NacionalCentro Nacional de Información Geográfica. <https:/www.ign.es/ign/layoutIn/ modeloDigitalTerreno.do> [consulta: julio 2016].

JANKOWSKI, P. (1995). "Integrating geographical information systems and multiple criteria decision-making methods». International Journal of Geographical Information Systems, 9 (3), 251-273.

<https://doi.org/10.1080/02693799508902036>

JIANG, H. y EASTMAN, R. (2000). "Application of fuzzy measures in multi-criteria evaluation in GIS». International Journal of Geographical Information Science, 14 (2), 173-184. <https://doi.org/10.1080/136588100240903>

KANG, S. y KIM, J-O. (2015). «Morphological analysis of green infrastructure in the Seoul metropolitan area, South Korea». Landscape and Ecological Engineering, 11, 259-268. <https://doi.org/10.1007/s11355-014-0268-5>

Kopperoinen, L.; ItKonen, P. y Niemelä, J. (2014). "Using expert knowledge in combining green infrastructure and ecosystem services in land use planning. An insight into a new place-based methodology». Landscape and Ecological Engineering, 29 (8), 1361-1375. <https://doi.org/10.1007/s10980-014-0014-2>

LAND Use Consultants (LUC) (2009). Green Infrastructure Guidance. Londres: Land Use Consultants (LUC), Natural England. <http://publications.naturalengland. org.uk/publication/35033> [consulta: enero 2015].

Liquete, C.; Kleeschulte, S.; Dige, G.; Maes, J.; Grizzetti, B.; Olah, B. y ZULIAN, G. (2015). "Mapping green infrastructure based on ecosystem services and ecological networks. A Pan-European case study». Environmental Science y Policy, 54, 268-280.

<https://doi.org/10.1016/j.envsci.2015.07.009>

López Romero, R. (2005). «Cálculo de rutas óptimas mediante SIG en el territorio de la ciudad celtibérica de Segeda. Propuesta metodológica». SALDVIE, 5 (2005), 95-111. <https://dialnet.unirioja.es/descarga/articulo/2293226.pdf> [consulta: junio 2016].

Machado, A.; Redondo, C. E. y Carralero, I. (2004). «Ensayando un índice de naturalidad en Canarias». En: J. M. Fernández-Palacios y C. Morici (eds.). 
Ecología insular/Island Ecology. Canarias: Asociación Española de Ecología Terrestre (AEET)-Cabildo Insular de La Palma, 413-438. <http://www.antoniomachado. net/wp-content/uploads/pdf/technical/2004-ensayando-un-indice-naturalidad-encanarias.pdf> [consulta: abril 2016].

MalCZEWSKI, J. (1999). GIS and Multicriteria Decision Analysis. Nueva York: John Wiley \& Sons, Inc.

Mallarach, J. M. (2004). Análisis y diagnóstico de la conectividad ecológica y paisajística en el sector sur del Anillo Verde de Vitoria-Gasteiz. Estudio de opciones y alternativas para maximizar el beneficio social y ecológico de los enlaces potenciales del Anillo Verde con los Montes de Vitoria. Vitoria-Gasteiz: Centro de Estudios Ambientales, Ayuntamiento de Vitoria-Gasteiz, 21 pp. <https://www.vitoria-gasteiz.org/wb021/ http/contenidosEstaticos/adjuntos/es/71/95/37195.pdf> [consulta: abril 2016].

Marull, J. y MallaraCH, J. M. (2005). «A GIS methodology for assessing ecological connectivity. Application to the Barcelona Metropolitan Area». Landscape and Urban Planning, 71, 243-262.

<https://doi.org/10.1016/j.landurbplan.2004.03.007>

- (2002). «La conectividad ecológica en el Área Metropolitana de Barcelona». Ecosistemas, 2002/2. <http://rua.ua.es/dspace/handle/10045/9814> [consulta: julio 2016].

MCGARIGAL, K. (2015). Fragstats help. Amherst: University of Massachusetts. <http:// www.umass.edu/landeco/research/fragstats/documents/fragstats.help.4.2.pdf> [consulta: julio 2016].

Natural England (2009). Green Growth for Green Communities. A selection of regional case studies. ParkCity Conference 2009. Londres: Natural England, 33 pp. <http://www.planethealthcymru.org/sitesplus/documents/886/green_growth_for_ green_communities.pdf> [consulta: julio 2016].

Ndubisi, F. (2003). Ecological Planning. A Historical and Comparative Synthesis. Baltimore: John Hopkins University Press, $304 \mathrm{pp}$.

Nijkamp, P. y Van Delft, A. (1977). Multi-Criteria Analysis and Regional Decision-Making. Leiden: Martinus Nijhoff.

Oñate, J. J.; Pereira, D.; Acebes, P. y García, E. (2014). Propuesta metodológica para identificación y valoración de Sistemas de Alto Valor Natural (Proyecto SAVN). Madrid: WWF-España. <http://awsassets.wwf.es/downloads/wwf_savn_propuesta_metodologica_def.pdf> [consulta: julio 2016].

Pla, M.; Brotons, L. y CAmpeny, R. (2007). "Obtención de mapas de conectividad funcional de vertebrados para su utilización en planificación territorial: aplicación en la planificación de espacios libres de la Provincia de Barcelona». VIII Congreso sobre SIG y Teledetección en Ecología, SIGTECO-8. Mérida (España), del 15 al 17 de febrero de 2007. <http:/www.minuartia.com/data/publicacions/bio/31080 91002_0701bioconnectividadsigtecoespminuartia.pdf> [consulta: junio 2016].

REMOlinA-AngariTA, F. (2011). «Figuras municipales de conservación ambiental en Colombia: ¿áreas protegidas, redes ecológicas o infraestructuras verdes?». Revista Nodo. Arquitectura, Ciudad, Medio Ambiente, 6 (11), 65-76. <http://dialnet.unirioja.es/servlet/articulo? codigo=3983371 $>$ [consulta: abril 2013].

Rodríguez Espinosa, V. M. y Aguilera Benavente, F. (2016). "¿Infraestructuras Verdes en la planificación territorial española?». Ciudad y Territorio. Estudios Territoriales-Ministerio de Fomento, 189, 399-418.

Rodríguez Espinosa, V. M.; Aguilera Benavente, F.; Gómez Delgado, M.; Salado García, M. J.; Lucas Olmo, L. y Cases Martínez, A. (2015). «Propuesta 
de Infraestructura Verde en un ámbito metropolitano. Aplicación al Corredor del Henares (Comunidad de Madrid-Guadalajara». En: J. DE LA RIVA; P. IBARRA; R. MONTORIO y M. RODRIGUES (eds.). Análisis espacial y representación geográfica: innovación y aplicación. Zaragoza: Universidad de Zaragoza-AGE, 383-392.

Romo Peña, C. y Torres Cantero, J. C. (2011). "Superficies de coste anisotrópicas». V Jornadas de SIG libre. Girona (España), 23-25 marzo 2011. Universitat de Girona: Servei de Sistemes d'Informació Geogràfica i Teledetecció-SIGTE. <http://www.sigte.udg.edu/jornadassiglibre2011/uploads/articulos/art35.pdf> [consulta: junio 2016].

Sbara, A.; Cueto Rúa, V.; Moroni, L.; Waslet, C. y Murace, P. (2007). «El pensamiento proyectual en la escala regional. El verde como infraestructura territorial». Pampa. Revista Interuniversitaria de Estudios Territoriales, 3, 91-101. <http://dialnet.unirioja.es/servlet/articulo?codigo=2756301> [consulta: abril 2013].

Soria-Lara, J. A.; Aguilera-Benavente, F. y Arranz-López, A. (2016). «Integrating land use and transport practice through spatial metrics». Transport Research, 91 (Part A), 330-345. <https://doi.org/10.1016/j.tra.2016.06.023>

Theobald, D. M.; Norman, J. B. y Sherburne, M. R. (2006). FunConn v1 User's Manual. ArcGIS tools for Functional Connectivity Modeling. Natural Resource Ecology Lab, Colorado State University. 17 de abril de 2006. 47 pp. <http://www.stat. colostate.edu/ nsu/starmap/learningmaterials/technicalreports/FunConn.Users. Guide.pdf> [consulta: junio 2016].

Town and Country Planning Association (TCPA) (2012). Planning for a Healthy Environment. Good Practice Guidance for Green Infrastructure and Biodiversity. Londres: Town and Country Planning Association and The Wildlife Trusts, $36 \mathrm{pp}$.

VogT, P. (2016). Guidos Toolbox (Graphical User Interface for the Description of image Objects and their Shapes). Digital image analysis software collection. <http://dx.doi.org/10.13140/RG.2.1.2633.8320>

Voogd, H. (1983). Multicriteria Evaluation for Urban and Regional Planning. Londres: Pion.

Wood, N. J. y Schmidtlein, M. C. (2012). «Anisotropic path modeling to assess pedestrian-evacuation potential from Cascadia-related tsunamis in the US Pacific Northwest». Natural Hazards, 62, 275-300. <https://doi.org/10.1007/s11069-011-9994-2> 